Bull. Austral. Math. Soc.

VoL. 73 (2006) [255-262]

\title{
REVERSE INEQUALITIES FOR THE NUMERICAL RADIUS OF LINEAR OPERATORS IN HILBERT SPACES
}

\section{S.S. Dragomir}

Some elementary inequalities providing upper bounds for the difference of the norm and the numerical radius of a bounded linear operator on Hilbert spaces under appropriate conditions are given.

\section{INTRODUCTION}

Let $(H ;\langle\cdot, \cdot\rangle)$ be a complex Hilbert space. The numerical range of an operator $T$ is the subset of the complex numbers $\mathbb{C}$ given by $[1$, p. 1$]$ :

$$
W(T)=\{\langle T x, x\rangle, x \in H,\|x\|=1\} .
$$

The following properties of $W(T)$ are immediate:

(i) $W(\alpha I+\beta T)=\alpha+\beta W(T)$ for $\alpha, \beta \in \mathbb{C}$;

(ii) $W\left(T^{*}\right)=\{\bar{\lambda}, \lambda \in W(T)\}$, where $T^{*}$ is the adjoint operator of $T$;

(iii) $W\left(U^{*} T U\right)=W(T)$ for any unitary operator $U$.

The following classical fact about the geometry of the numerical range $[1$, p. 4] may be stated:

THEOREM 1. (Toeplitz-Hausdorff.) The numerical range of an operator is convex.

An important use of $W(T)$ is to bound the spectrum $\sigma(T)$ of the operator $T[1$, p. 6$]$ :

THEOREM 2. (Spectral inclusion.) The spectrum of an operator is contained in the closure of its numerical range.

The self-adjoint operators have their spectra bounded sharply by the numerical range $[1$, p. 7]:

THEOREM 3. The following statements hold true:

(i) $T$ is self-adjoint if and only if $W(T)$ is real;

(ii) If $T$ is self-adjoint and $W(T)=[m, M]$ (the closed interval of real numbers $m, M)$, then $\|T\|=\max \{|m|,|M|\}$.

Received 15th November, 2005

The author would like to thank the referee for his/her suggestions that have been implemented in the final version of the paper.

Copyright Clearance Centre, Inc. Serial-fee code: 0004-9727/06 \$A2.00+0.00. 
(iii) If $W(T)=[m, M]$, then $m, M \in \sigma(T)$.

The numerical radius $w(T)$ of an operator $T$ on $H$ is given by $[1$, p. 8]:

$$
w(T)=\sup \{|\lambda|, \lambda \in W(T)\}=\sup \{|\langle T x, x\rangle|,\|x\|=1\} .
$$

Obviously, by (1.1), for any $x \in H$ one has

$$
|\langle T x, x\rangle| \leqslant w(T)\|x\|^{2} .
$$

It is well known that $w(\cdot)$ is a norm on the Banach algebra $B(H)$ of all bounded linear operators $T: H \rightarrow H$, that is,

(i) $w(T) \geqslant 0$ for any $T \in B(H)$ and $w(T)=0$ if and only if $T=0$;

(ii) $w(\lambda T)=|\lambda| w(T)$ for any $\lambda \in \mathbb{C}$ and $T \in B(H)$;

(iii) $w(T+V) \leqslant w(T)+w(V)$ for any $T, V \in B(H)$.

This norm is equivalent with the operator norm. In fact, the following more precise result holds [1, p. 9]:

ThEOREM 4. (Equivalent norm.) For any $T \in B(H)$ one has

$$
w(T) \leqslant\|T\| \leqslant 2 w(T) .
$$

Let us now look at two extreme cases of the inequality (1.3). In the following $r(t):=\sup \{|\lambda|, \lambda \in \sigma(T)\}$ will denote the spectral radius of $T$ and $\sigma_{p}(T)=\{\lambda$ $\in \sigma(T), T f=\lambda f$ for some $f \in H\}$ the point spectrum of $T$.

The following results hold $[1$, p. 10]:

THEOREM 5 . We have

(i) If $w(T)=\|T\|$, then $r(T)=\|T\|$.

(ii) If $\lambda \in W(T)$ and $|\lambda|=\|T\|$, then $\lambda \in \sigma_{p}(T)$.

To address the other extreme case $w(T)=\|T\| / 2$, we can state the following suffcient condition in terms of (see $[1$, p. 11])

$$
R(T):=\{T f, f \in H\} \text { and } R\left(T^{*}\right):=\left\{T^{*} f, f \in H\right\} .
$$

THEOREM 6. If $R(T) \perp R\left(T^{*}\right)$, then $w(T)=\|T\| / 2$.

It is well-known that the two-dimensional shift

$$
S_{2}=\left[\begin{array}{ll}
0 & 0 \\
1 & 0
\end{array}\right]
$$

has the property that $w(T)=\|T\| / 2$.

The following theorem shows that some operators $T$ with $w(T)=\|T\| / 2$ have $S_{2}$ as a component $[1$, p. 11$]$ : 
THEOREM 7. If $w(T)=\|T\| / 2$ and $T$ attains its norm, then $T$ has a twodimensional reducing subspace on which it is the shift $S_{2}$.

For other results on numerical radius, see [2, Chapter 11].

The main aim of the present paper is to point out some upper bounds for the nonnegative difference

$$
\|T\|-w(T) \quad\left(\|T\|^{2}-(w(T))^{2}\right)
$$

under appropriate assumptions for the bounded linear operator $T: H \rightarrow H$.

\section{The Results}

The following results may be stated:

THEOREM 8. Let $T: H \rightarrow H$ be a bounded linear operator on the complex Hilbert space $H$. If $\lambda \in \mathbb{C} \backslash\{0\}$ and $r>0$ are such that

$$
\|T-\lambda I\| \leqslant r,
$$

where $I: H \rightarrow H$ is the identity operator on $H$, then

$$
(0 \leqslant)\|T\|-w(T) \leqslant \frac{1}{2} \cdot \frac{r^{2}}{|\lambda|} .
$$

PROOF: For $x \in H$ with $\|x\|=1$, we have from (2.1) that

$$
\|T x-\lambda x\| \leqslant\|T-\lambda I\| \leqslant r
$$

giving

$$
\|T x\|^{2}+|\lambda|^{2} \leqslant 2 \operatorname{Re}[\lambda\langle T x, x\rangle]+r^{2} \leqslant 2|\lambda||\langle T x, x\rangle|+r^{2} .
$$

Taking the supremum over $x \in H,\|x\|=1$ in (2.3) we get the following inequality that is of interest in itself:

$$
\|T\|^{2}+|\lambda|^{2} \leqslant 2 w(T)|\lambda|+r^{2} .
$$

Since, obviously,

$$
\|T\|^{2}+|\lambda|^{2} \geq 2\|T\||\lambda|,
$$

hence by (2.4) and (2.5) we deduce the desired inequality (2.2).

REMARK 1 . If the operator $T: H \rightarrow H$ is such that $R(T) \perp R\left(T^{*}\right),\|T\|=1$ and $\|T-I\| \leqslant 1$, then the equality holds in (2.2). Indeed, by Theorem 6 , we have in this case $w(T)=\|T\| / 2=1 / 2$ and since we can choose $\lambda=1, r=1$ in Theorem 8 , then we get in both sides of (2.2) the same quantity $1 / 2$. 
Problem 1. Find bounded linear operators $T: H \rightarrow H$ with $\|T\|=1, R(T) \perp R\left(T^{*}\right)$ and $\|T-\lambda I\| \leqslant|\lambda|^{1 / 2}$.

The following corollary may be stated:

Corollary 1. Let $A: H \rightarrow H$ be a bounded linear operator and $\varphi, \psi \in \mathbb{C}$ with $\psi \notin\{-\varphi, \varphi\}$. If

$$
\operatorname{Re}\langle\psi x-A x, A x-\varphi x\rangle \geqslant 0 \quad \text { for any } \quad x \in H,\|x\|=1
$$

then

$$
(0 \leqslant)\|A\|-w(A) \leqslant \frac{1}{4} \cdot \frac{|\psi-\varphi|^{2}}{|\psi+\varphi|} .
$$

Proof: Utilising the fact that in any Hilbert space the following two statements are equivalent:

(i) $\operatorname{Re}\langle u-x, x-z\rangle \geqslant 0, x, z, u \in H$;

(ii) $\|x-(z+u) / 2\| \leqslant\|u-z\| / 2$,

we deduce that $(2.6)$ is equivalent to

$$
\left\|A x-\frac{\psi+\varphi}{2} \cdot I x\right\| \leqslant \frac{1}{2}|\psi-\varphi|
$$

for any $x \in H,\|x\|=1$, which in its turn is equivalent with the operator norm inequality:

$$
\left\|A-\frac{\psi+\varphi}{2} \cdot I\right\| \leqslant \frac{1}{2}|\psi-\varphi| \text {. }
$$

Now, applying Theorem 8 for $T=A, \lambda=(\varphi+\psi) / 2$ and $r=|\varphi-\psi| / 2$, we deduce the desired result (2.7).

REMARK 2. Following [1, p. 25], we say that an operator $B: H \rightarrow H$ is accretive, if $\operatorname{Re}\langle B x, x\rangle \geqslant 0$ for any $x \in H$. One may observe that the assumption (2.6) above is then equivalent with the fact that the operator $\left(A^{*}-\bar{\varphi} I\right)(\psi I-A)$ is accretive.

Perhaps a more convenient sufficient condition in terms of positive operators is the following one:

Corollary 2. Let $\varphi, \psi \in \mathbb{C}$ with $\psi \notin\{-\varphi, \varphi\}$ and $A: H \rightarrow H$ a bounded linear operator in $H$. If $\left(A^{*}-\bar{\varphi} I\right)(\psi I-A)$ is self-adjoint and

$$
\left(A^{*}-\bar{\varphi} I\right)(\psi I-A) \geqslant 0
$$

in the operator partial order, then

$$
(0 \leqslant)\|A\|-w(A) \leqslant \frac{1}{4} \cdot \frac{|\psi-\varphi|^{2}}{|\psi+\varphi|} .
$$


COROLlary 3. Assume that $T, \lambda, r$ are as in Theorem 8. If, in addition,

$$
|| \lambda|-w(T)| \geqslant \rho,
$$

for some $\rho \geqslant 0$, then

$$
(0 \leqslant)\|T\|^{2}-w^{2}(T) \leqslant r^{2}-\rho^{2} .
$$

Proof: From (2.4) of Theorem 8, we have

$$
\begin{aligned}
\|T\|^{2}-w^{2}(T) & \leqslant r^{2}-w^{2}(T)+2 w(T)|\lambda|-|\lambda|^{2} \\
& =r^{2}-(|\lambda|-w(T))^{2} .
\end{aligned}
$$

The desired inequality follows from (2.12).

REMARK 3. In particular, if $\|T-\lambda I\| \leqslant r$ and $|\lambda|=w(T), \lambda \in \mathbb{C}$, then

$$
(0 \leqslant)\|T\|^{2}-w^{2}(T) \leqslant r^{2} .
$$

The following result may be stated as well.

THEOREM 9. Let $T: H \rightarrow H$ be a nonzero bounded linear operator on $H$ and $\lambda \in \mathbb{C} \backslash\{0\}, r>0$ with $|\lambda|>r$. If

$$
\|T-\lambda I\| \leqslant r
$$

then

$$
\sqrt{1-\frac{r^{2}}{|\lambda|^{2}}} \leqslant \frac{w(T)}{\|T\|} \quad(\leqslant 1) .
$$

Proof: From (2.4) of Theorem 8, we have

$$
\|T\|^{2}+|\lambda|^{2}-r^{2} \leqslant 2|\lambda| w(T),
$$

which implies, on dividing with $\sqrt{|\lambda|^{2}-r^{2}}>0$ that

$$
\frac{\|T\|^{2}}{\sqrt{|\lambda|^{2}-r^{2}}}+\sqrt{|\lambda|^{2}-r^{2}} \leqslant \frac{2|\lambda| w(T)}{\sqrt{|\lambda|^{2}-r^{2}}} .
$$

By the elementary inequality

$$
2\|T\| \leqslant \frac{\|T\|^{2}}{\sqrt{|\lambda|^{2}-r^{2}}}+\sqrt{|\lambda|^{2}-r^{2}}
$$

and by (2.18) we deduce

$$
\|T\| \leqslant \frac{w(T)|\lambda|}{\sqrt{|\lambda|^{2}-r^{2}}}
$$

which is equivalent to (2.17). 
REMARK 4. Squaring (2.17), we get the inequality

$$
(0 \leqslant)\|T\|^{2}-w^{2}(T) \leqslant \frac{r^{2}}{|\lambda|^{2}}\|T\|^{2} .
$$

REMARK 5. For any bounded linear operator $T: H \rightarrow H$ we have the relation $w(T)$ $\geqslant\|T\| / 2$. Inequality (2.17) would produce a refinement of this classic fact only in the case when

$$
\frac{1}{2} \leqslant\left(1-\frac{r^{2}}{|\lambda|^{2}}\right)^{1 / 2}
$$

which is equivalent to $r /|\lambda| \leqslant \sqrt{3} / 2$.

The following corollary holds.

Corollary 4. Let $\varphi, \psi \in \mathbb{C}$ with $\operatorname{Re}(\psi \bar{\varphi})>0$. If $T: H \rightarrow H$ is a bounded linear operator such that either (2.6) or (2.10) holds true, then:

$$
\frac{2 \sqrt{\operatorname{Re}(\psi \bar{\varphi})}}{|\psi+\varphi|} \leqslant \frac{w(T)}{\|T\|}(\leqslant 1)
$$

and

$$
(0 \leqslant)\|T\|^{2}-w^{2}(T) \leqslant\left|\frac{\psi-\varphi}{\psi+\varphi}\right|^{2}\|T\|^{2} .
$$

PROOF: If we consider $\lambda=(\psi+\varphi) / 2$ and $r=|\psi-\varphi| / 2$, then

$$
|\lambda|^{2}-r^{2}=|(\psi+\varphi) / 2|^{2}-|(\psi-\varphi) / 2|^{2}=\operatorname{Re}(\psi \bar{\varphi})>0 .
$$

Now, on applying Theorem 9, we deduce the desired result.

REMARK 6. If $|\psi-\varphi| \leqslant(\sqrt{3} / 2)|\psi+\varphi|, \operatorname{Re}(\psi \bar{\varphi})>0$, then (2.21) is a refinement of the inequality $w(T) \geqslant\|T\| / 2$.

The following result may be of interest as well.

THEOREM 10. Let $T: H \rightarrow H$ be a nonzero bounded linear operator on $H$ and $\lambda \in \mathbb{C} \backslash\{0\}, r>0$ with $|\lambda|>r$. If

$$
\|T-\lambda I\| \leqslant r
$$

then

$$
(0 \leqslant)\|T\|^{2}-w^{2}(T) \leqslant \frac{2 r^{2}}{|\lambda|+\sqrt{|\lambda|^{2}-r^{2}}} w(T) .
$$

Proof: From the proof of Theorem 8, we have

$$
\|T x\|^{2}+|\lambda|^{2} \leqslant 2 \operatorname{Re}[\lambda\langle T x, x\rangle]+r^{2}
$$


for any $x \in H,\|x\|=1$.

If we divide (2.25) by $|\lambda||\langle T x, x\rangle|$, (which, by (2.25), is positive) then we obtain

$$
\frac{\|T x\|^{2}}{|\lambda||\langle T x, x\rangle|} \leqslant \frac{2 \operatorname{Re}[\bar{\lambda}\langle T x, x\rangle]}{|\lambda||\langle T x, x\rangle|}+\frac{r^{2}}{|\lambda||\langle T x, x\rangle|}-\frac{|\lambda|}{|\langle T x, x\rangle|}
$$

for any $x \in H,\|x\|=1$.

If we subtract in (2.26) the same quantity $|\langle T x, x\rangle| /|\lambda|$ from both sides, then we get

$$
\begin{aligned}
& \frac{\|T x\|^{2}}{\overline{\lambda\|\||(T x, x\rangle|}-\frac{|\langle T x, x\rangle|}{|\lambda|}} \\
& \quad \leqslant \frac{2 \operatorname{Re}[\bar{\lambda}\langle T x, x t\rangle]}{|\lambda||\langle T x, x\rangle|}+\frac{r^{2}}{|\lambda||\langle x, x\rangle|}-\frac{|\langle T x, x\rangle|}{|\lambda|}-\frac{|\lambda|}{|\langle T x, x\rangle|} \\
& \quad=\frac{2 \operatorname{Re}[\bar{\lambda}\langle T x, x\rangle]}{|\lambda||\langle T x, x\rangle|}-\frac{|\lambda|^{2}-r^{2}}{|\lambda||\langle x, x\rangle|}-\frac{|\langle T x, x\rangle|}{|\lambda|} \\
& \quad=\frac{2 \operatorname{Re}[\bar{\lambda}\langle T x, x\rangle]}{|\lambda||\langle T x, x\rangle|}-\left(\frac{\sqrt{|\lambda|^{2}-r^{2}}}{\sqrt{|\lambda||\langle T x, x\rangle|}}-\frac{\sqrt{|\langle T x, x\rangle|}}{\sqrt{|\lambda|}}\right)^{2}-2 \frac{\sqrt{|\lambda|^{2}-r^{2}}}{|\lambda|}
\end{aligned}
$$

Since

$$
\operatorname{Re}[\lambda\langle T x, x\rangle] \leqslant|\lambda||\langle T x, x\rangle|
$$

and

$$
\left(\frac{\sqrt{|\lambda|^{2}-r^{2}}}{\sqrt{|\lambda||\langle\bar{T} x, x\rangle|}}-\frac{\sqrt{|\langle T x, x\rangle|}}{\sqrt{|\lambda|}}\right)^{2} \geqslant 0
$$

by (2.27) we get

which gives the inequality

$$
\frac{\|T x\|^{2}}{|\lambda||(T x, x\rangle|}-\frac{|\langle T x, x\rangle|}{|\lambda|} \leqslant \frac{2\left(|\lambda|-\sqrt{|\lambda|^{2}-r^{2}}\right)}{|\lambda|}
$$

$$
\|T x\|^{2} \leqslant|\langle T x, x\rangle|^{2}+2|\langle T x, x\rangle|\left(|\lambda|-\sqrt{|\lambda|^{2}-r^{2}}\right)
$$

for any $x \in H,\|x\|=1$.

Taking the supremum over $x \in H,\|x\|=1$, we get

$$
\begin{aligned}
\|T\|^{2} & \leqslant \sup \left\{|\langle T x, x\rangle|^{2}+2|\langle T x, x\rangle|\left(|\lambda|-\sqrt{|\lambda|^{2}-r^{2}}\right)\right\} \\
& \leqslant \sup \left\{|\langle T x, x\rangle|^{2}\right\}+2\left(|\lambda|-\sqrt{|\lambda|^{2}-r^{2}}\right) \sup \{|\langle T x, x\rangle|\} \\
& =w^{2}(T)+2\left(|\lambda|-\sqrt{|\lambda|^{2}-r^{2}}\right) w(T),
\end{aligned}
$$

which is clearly equivalent to (2.24).

Corollary 5. Let $\varphi, \psi \in \mathbb{C}$ with $\operatorname{Re}(\psi \bar{\varphi})>0$. If $A: H \rightarrow H$ is a bounded linear operator such that either (2.6) or (2.10) hold true, then:

$$
(0 \leqslant)\|A\|^{2}-w^{2}(A) \leqslant[|\psi+\varphi|-2 \sqrt{\operatorname{Re}(\psi \bar{\varphi})}] w(A) .
$$


REMARK 7. If $M \geqslant m>0$ are such that either $\left(A^{*}-m I\right)(M I-A)$ is accretive, or, sufficiently, $\left(A^{*}-m I\right)(M I-A)$ is self-adjoint and

$$
\left(A^{*}-m I\right)(M I-A) \geqslant 0 \text { in the operator partial order, }
$$

then, by (2.21) we have:

$$
(1 \leqslant) \frac{\|A\|}{w(A)} \leqslant \frac{M+m}{2 \sqrt{m M}}
$$

which is equivalent to

$$
(0 \leqslant)\|A\|-w(A) \leqslant \frac{(\sqrt{M}-\sqrt{m})^{2}}{2 \sqrt{m M}} w(A),
$$

while from (2.24) we have

$$
(0 \leqslant)\|A\|^{2}-w^{2}(A) \leqslant(\sqrt{M}-\sqrt{m})^{2} w(A) .
$$

Also, the inequality (2.7) becomes

$$
(0 \leqslant)\|A\|-w(A) \leqslant \frac{1}{4} \cdot \frac{(M-m)^{2}}{M+m} .
$$

\section{REFERENCES}

[1] K.E. Gustafson and D.K.M. Rao, Numerical range (Springer-Verlag, New York, 1997).

[2] P.R. Halmos, A Hilbert space problem book, (Second edition) (Springer-Verlag, New York, Heidelberg, Berlin, 1982).

School of Computer Science and Mathematics

Victoria University of Technology

PO Box 14428

Melbourne City, Vic. 8001

Australia

e-mail: sever.dragomir@vu.edu.au 